\title{
HERMITE SERIES AS BOUNDARY VALUES
}

\author{
BY
}

\author{
G. G. WALTER
}

\begin{abstract}
The relations between Hermite series expansions of functions and tempered distributions on the real axis and holomorphic or harmonic functions or generalizations of them in the upper half plane are studied. The Hermite series expansions of $H^{2}$ functions are characterized in terms of their coefficients. Series of analytic representations of Hermite functions, series of Hermite functions of the second kind, and combined series of Hermite functions of the first and second kind are investigated. The functions to which these series converge in the upper half plane are shown to approach (in various ways) the distributions or functions whose Hermite series have the same coefficients.
\end{abstract}

1. Introduction. Much of the theory of wave motion in classical physics is based on solutions to boundary value problems associated with the equation $y^{\prime \prime}=-\lambda y$, the harmonic oscillator equation. When one moves to quantum mechanics, the fundamental equation of wave motion has the form $y^{\prime \prime}-x^{2} y=-\lambda y$. The eigenfunctions of the former are the trigonometric functions and of the latter are the Hermite functions. The theory of trigonometric Fourier series has been a well-known and widely used part of the development of the theory of classical wave motion. The theory of Hermite Fourier series is little used apart from the basic oscillator. One might expect that a further development of the theory of Hermite series along the lines of that of trigonometric series would lead to new insight.

Trigonometric Fourier series are closely connected with boundary values of holomorphic and harmonic functions in the unit disk $D$. The various relations have been extensively studied and are quite well known.

If we move to the real line, no parallel theory based on series seems to exist. The analogous results for the upper and lower half plane are usually based on integral operators. A natural orthonormal sequence to use on the real line is the same sequence of Hermite functions $\left\{h_{n}\right\}$. The only attempt to derive analogous results that I know of is the work of Muckenhoupt [M], whose approach differs considerably from the one we shall follow here. We shall introduce functions related to these Hermite functions whose behavior off the real axis emulates some aspects of the behavior of the functions $r^{ \pm n} e^{i n \theta}$ and $e^{i n z}$ off the boundary

Received by the editors March 19, 1974 and, in revised form, September 25, 1974.

AMS (MOS) subject classifications (1970). Primary 33A65; Secondary 30A78.

Key words and phrases. Harmonic oscillator, Hermite functions, harmonic functions, tempered distributions, boundary values. 
of $D$. This is necessary since the behavior of the $h_{n}$ is very bad off the real axis; series of $h_{n}$, all of whose derivatives converge uniformly on the real axis, may fail to be even pointwise convergent off the axis.

We shall discuss four different approaches each of which imitates some aspects of trigonometric series. The first approach is based on sequences of coefficients of Hermite series, the second on functions which are analytic representations of Hermite functions, and the last two on Hermite functions of the second kind.

2. Hermite expansions of $H^{p}$ functions. In the case of trigonometric Fourier series, those belonging to $H^{p}$ functions are distinguised by having their negative coefficients equal to zero. No such simple conditions hold for Hermite series of functions on $R^{1}$. Indeed, none of the Hermite functions themselves belong to $H^{p}$ of either the upper or lower half plane since the Fourier transform of such $H^{p}$ functions must vanish on a half line. The Fourier transform of $h_{n}$ \{i.e. $(-i)^{n} h_{n}$ \} does not. In order to determine which sequences of coefficients do belong to functions in $H^{p}$, we use the auxilliary sequences $\left\{\alpha_{n k}\right\}$ where

$$
\alpha_{n k}=\int_{0}^{\infty} h_{n} h_{k}, \quad n, k=0,1, \ldots
$$

Then $\Sigma i^{n} \alpha_{n k} h_{n}$ is the Hermite series of a function in $H^{2}$ of the upper half plane since its Fourier transform vanishes on $(-\infty, 0)$. By using the recurrence formulae of the Hermite functions we may derive the following properties of the $\alpha_{n k}$ :

$$
\begin{gathered}
\alpha_{n k}=1 / 2 \delta_{n k}, \quad n+k \text { even, } \\
\sum_{n=0}^{\infty} \alpha_{k n} \alpha_{n l}=\alpha_{k l}, \\
\alpha_{2 n+1,2 k}=\frac{(-1)^{n-k}(2 n+1) !(2 k) !}{(2 n-2 k+1) 2 \pi 2^{n} n ! 2^{k} k !} .
\end{gathered}
$$

These furnish the basic tools to prove

THEOREM 1. Let $\Sigma c_{n} h_{n}$ be the Hermite series expansion of a function $f \in L^{2}(-\infty, \infty)$; let

$$
\begin{aligned}
a_{n}=\sum_{k=0}^{\infty} i^{(n-k)} \alpha_{n k} c_{k}, \quad n & =0,1, \ldots, \\
\left(b_{n}\right. & \left.=\sum_{k=0}^{\infty}(-i)^{n-k} \alpha_{n k} c_{k}, \quad n=0,1, \ldots\right) ;
\end{aligned}
$$

then $\Sigma a_{n} h_{n}\left(\Sigma b_{n} h_{n}\right)$ is the expansion of a function in $H^{2}$ of the upper half (lower half) plane. Moreover $c_{n}=a_{n}+b_{n}$, and if $f$ itself $\in H^{2}$ of the upper half plane, $c_{n}=a_{n}$. 
The proof involves using the infinite matrices $A=\left[i^{n-k} \alpha_{n k}\right]$ and $\bar{A}=$ $\left[(-i)^{n-k} \alpha_{n k}\right]$ as operators on $l^{2}$. They have the properties that $A+\bar{A}=I$ and $A \bar{A}=0$. From these and the fact that $A$ maps $l^{2}$ into sequences which are the Hermite coefficients of $H^{2}$ functions, the conclusions follow.

Unfortunately, when we look at convergence of Hermite expansions of $H^{2}$ functions, we cannot in general extend the convergence on the real axis to the complex plane. In order to imitate the convergence inside the unit disk of Fourier series of $H^{2}$ functions we need to introduce new functions associated with the Hermite functions. One such family of functions is studied in the next section.

3. Series of projections of Hermite functions. Since the Hermite functions themselves are not in $\mathrm{H}^{2}$ and since Hermite series of $\mathrm{H}^{2}$ functions do not behave very well off the real axis, it is better to consider other series instead. In this section we shall consider the projections of the Hermite functions on $H^{2}$ and the behavior of series of the projections in the complex plane. These projections $\left\{g_{k}\right\}$ may be given by the functions

$$
g_{k}(t)=\sum_{n=0}^{\infty}(i)^{n-k} \alpha_{n k} h_{n}(t), \quad k=0,1,2, \ldots
$$

Then if $f \in H^{2}$ with Hermite expansion $\Sigma a_{n} h_{n}$ we see that

$$
f=\sum_{n} a_{n} h_{n}=\sum_{n}\left\{\sum_{k}(i)^{n-k} \alpha_{n k} a_{k}\right\} h_{n}=\sum_{n}\left\{\int f \overline{g_{n}}\right\} h_{n}
$$

by Parseval's equality since by the symmetry of $\alpha_{n k}$, the Hermite coefficients of $\overline{g_{n}}$ are $i^{n-k} \alpha_{n k}$. Thus we find that $a_{n}=\int f h_{n}=\int f \overline{g_{n}}$ for $f \in H^{2}$ of the upper half plane $\left(C^{+}\right)$and similarly $\int f h_{n}=\int f g_{n}$ for $f \in H^{2}$ of the lower half plane

$\left(C^{-}\right)$. Whenever $f \in L^{2}$ we can rewrite the results of Theorem 1 as

$$
\int f h_{n}=\int f \bar{g} g_{n}+\int f g_{n} .
$$

Whence it follows that

$$
\overline{g_{n}}+g_{n}=h_{n} \quad \text { a.e. }
$$

Thus all $f \in L^{2}$ may be written as

$$
f=\sum c_{n} h_{n}=\sum c_{n} g_{n}+\sum c_{n} \overline{g_{n}}
$$

where $\Sigma c_{n} g_{n} \in H^{2}\left(C^{+}\right)$. There $\left\{g_{n}\right\}$ may be shown, by using the properties of the $\alpha_{n k}$, to satisfy

$$
\begin{gathered}
g_{k}(x)=\frac{1}{\sqrt{2 \pi}} \int_{0}^{\infty} e^{i x t}(-i)^{k} h_{k}(t) d t \\
g_{k}(z)=\frac{1}{2 \pi i} \int_{-\infty}^{\infty} \frac{h_{k}(x)}{x-z} d x, \quad \operatorname{Im} z>0
\end{gathered}
$$


Using these properties we may attack the problem of convergence in the complex plane.

THEOREM 2. Let $f \in L^{2}(-\infty, \infty)$ with Hermite series expansion $\Sigma c_{n} h_{n}$; let

$$
f^{+}(z)=\frac{1}{2 \pi i} \int_{-\infty}^{\infty} \frac{f(x)}{x-z} d x
$$

be the projection of $f$ on $H^{2}, \operatorname{Im} z>0$; then $\Sigma c_{n} g_{n}(z)$

(i) converges absolutely for $\operatorname{Im} z>0$,

(ii) converges uniformly to $f^{+}(z)$ in any region for which $\operatorname{Im} z \geqslant \epsilon>0$,

(iii) converges in the sense of $L^{2}(-\infty, \infty)$ for each fixed $y \geqslant 0$, and $\Sigma c_{n}\left\{\bar{g}_{n}(z)+g_{n}(z)\right\}$ converges to a harmonic function whose boundary values on $(-\infty, \infty)$ are given by $f(x)$.

The first conclusion follows from Bessel's inequality since the $g_{n}(z)$ are just the Hermite coefficients of $(2 \pi i)^{-1}(x-z)^{-1}$. Hence we see that

$$
\left|\sum_{n=0}^{N}\right| c_{n} g_{n}(z)||^{2} \leqslant \sum\left|c_{n}\right|^{2} \sum\left|g_{n}(z)\right|^{2} \leqslant\|f\|_{2}^{2} \int_{-\infty}^{\infty} \frac{1}{4 \pi^{2}|x-z|^{2}} d x .
$$

To prove the uniform convergence we consider the equality

$$
\begin{aligned}
\left|\sum_{n=0}^{N} c_{n} g_{n}(z)-f^{+}(z)\right| & =\frac{1}{2 \pi}\left|\int_{-\infty}^{\infty} \frac{\Sigma_{n=0}^{N} c_{n} h_{n}-f}{x-z} d x\right| \\
& \leqslant\left\|\sum_{n=0}^{N} c_{n} h_{n}-f\right\|_{2}\left\{\frac{1}{2 \sqrt{\pi y}}\right\}
\end{aligned}
$$

from which our assertion follows immediately.

Finally to show $L^{2}$ convergence we use Plancherel's identity twice to calculate

$$
\begin{aligned}
\int_{-\infty}^{\infty}\left|\sum_{n=0}^{N} c_{n} g_{n}(x+i y)-f^{+}(x+i y)\right|^{2} d x \\
\quad=\int_{0}^{\infty} e^{-2 y t}\left|\sum_{n=0}^{N} c_{n} h_{n}(t)(-i)^{n}-\hat{f}(t)\right|^{2} d t \\
\quad \leqslant \int_{-\infty}^{\infty}\left|\sum_{n=0}^{N} c_{n} h_{n}(t)(-i)^{n}-\hat{f}(t)\right|^{2} d t \\
=\left\|\sum_{n=0}^{N} c_{n} h_{n}-f\right\|_{2}^{2} \rightarrow 0 \text { as } N \rightarrow \infty .
\end{aligned}
$$

Here we have used the fact that 


$$
f^{+}(x+i y)=\frac{1}{2 \pi i} \int_{0}^{\infty} e^{i(x+i y) t} \hat{f}(t) d t
$$

which is another consequence of Plancherel's identity. The last statement follows immediately from the others.

There are still many convergence problems that we have left unresolved. For example we have not looked at the problems associated with $H^{p}$ spaces for $p \neq 2$. In particular the questions associated with $H^{1}$ are difficult. We could also look at pointwise convergence on the real axis at, say, points of smoothness of $f$.

We note that with the replacement of $h_{n}$ by $g_{n}$ the convergence of the series is improved off the real axis. However it is still not good enough to include a number of cases of interest. It is well known that each function of polynomial growth has a Hermite series with coefficients $O\left(n^{p}\right)$ convergent to it in the sense of (tempered) distributions. Also each such function has an analytic representation to which we should like the series with the $g_{n}$ to converge with some sort of uniformity. Therefore, in order to improve the convergence we introduce another related sequence of functions $\left\{\widetilde{h}_{n}\right\}$ the Hermite functions of the second kind. These are more closely analogous to the functions $\left\{e^{i n z}\right\}$ on the unit circle.

4. Expansions in series of Hermite functions of the second kind. These Hermite functions of the second kind $\widetilde{h}_{n}$ are solutions to the Hermite equation

$$
\left(z^{2}-D^{2}\right) w=(2 n+1) w, \quad n=0,1, \ldots,
$$

which vanish at $i_{\infty}$ when $z$ is in the upper half plane and at $-i_{\infty}$ when $z$ is in the lower half plane. The functions were studied in [W] where it is shown that they satisfy the same recurrence formulae as the Hermite functions themselves, as well as the following formulae:

$$
\begin{gathered}
\tilde{h}_{n}(z)=h_{n}(z) \int_{i \infty}^{z} \frac{2}{h_{n}^{2}}, \quad \operatorname{Im} z>0 . \\
\tilde{h}_{n}(z) h_{k}(z)=-\int_{-\infty}^{\infty} \frac{h_{n}(x) h_{k}(x)}{x-z} d x, \quad \operatorname{Im} z \neq 0, n \geqslant k, \\
\tilde{h}_{n}(x+i 0)-\tilde{h}_{n}(x-i 0)=-2 \pi i h_{n}(x) .
\end{gathered}
$$

THEOREM 3. Let $f$ be a continuous real valued function of polynomial growth with Hermite series expansion $\Sigma c_{n} h_{n}$; then

(i) $\Sigma\left(i c_{n} / 2 \pi\right) \tilde{h}_{n}(z)$ converges uniformly in compact subsets of either the upper or lower open half plane,

(ii) $\Sigma\left(i c_{n} / 2 \pi\right)\left\{\widetilde{h}_{n}(z)-\widetilde{h}_{n}(\bar{z})\right\}$ converges in the upper half plane to a real harmonic function $u(z)$,

(iii) $\lim _{y \rightarrow 0} u(x+i y)=f(x)$ uniformly on compact sets of $R$. 
It is well known that a function of polynomial growth belongs to $S^{\prime}$, the space of tempered distributions; convergence in $S^{\prime}$ is equivalent to weak convergence, i.e. $\Sigma c_{n} h_{n}$ converges as $\Sigma c_{n}\left\langle h_{n}, \varphi\right\rangle$ converges for each $\varphi \in S$. Also an element $f$ of $S^{\prime}$ has a Hermite expansion $\Sigma c_{n} h_{n}$ whose coefficients $c_{n}=O\left(n^{p}\right)$ for some integer $p$, and which converges to $f$. Now by property (ii)

$$
\begin{aligned}
\sum \frac{i c_{n}}{2 \pi} \widetilde{h}_{n}(z) & =\sum \frac{i c_{n}}{2 \pi} \int_{-\infty}^{\infty} \frac{h_{n}(t) \exp \left(z^{2} / 2\right) \exp \left(-t^{2} / 2\right)}{z-t} d t \\
& =\exp \left(z^{2} / 2\right) \sum c_{n} \frac{1}{2 \pi i} \int_{-\infty}^{\infty} \frac{h_{n}(t)}{t-z} \exp \left(-t^{2} / 2\right) d t \\
& =\exp \left(z^{2} / 2\right) \sum c_{n}\left\langle\varphi_{z}, h_{n}\right\rangle
\end{aligned}
$$

where $\varphi_{z}(t)=\exp \left(-t^{2} / 2\right) /(t-z) \in S$. Therefore this series converges pointwise for $\operatorname{Im} z \neq 0$.

To show this convergence is in fact uniform on compact sets we use the fact that $h_{n}=\left(x^{2}-D^{2}\right)^{p+2} h_{n} /(2 n+1)^{p+2}$, where $p$ is the nonnegative integer associated with the asymptotic behavior of the coefficients. Then we have

$$
\sum c_{n}\left\langle\varphi_{z}, \frac{\left(x^{2}-D^{2}\right)^{p+2}}{(2 n+1)^{p+2}} h_{n}\right\rangle=\sum \frac{c_{n}}{(2 n+1)^{p+2}}\left\langle\left(x^{2}-D^{2}\right)^{p+2} \varphi_{z}, h_{n}\right\rangle
$$

The series $\Sigma\left(c_{n} /(2 n+1)^{p+2}\right)$ converges absolutely and $\left\langle\left(x^{2}-D^{2}\right)^{p+2} \varphi_{z}, h_{n}\right\rangle$ is uniformly bounded on compact sets avoiding the real axis.

The convergence in (ii) follows from that in (i), and the limit function will clearly be harmonic. We need only show that it be real. This can be done by showing $i\left(\widetilde{h}_{n}(z)-\widetilde{h}_{n}(\bar{z})\right)$ to be real. But we have, by (17), with $k=0$,

$$
i\left(\widetilde{h}_{n}(z)-\widetilde{h}_{n}(\bar{z})\right)=-i \int_{-\infty}^{\infty} h_{n}(t) \exp \left(-t^{2} / 2\right)\left\{\frac{\exp \left(z^{2} / 2\right)}{t-z}-\frac{\exp \left(\bar{z}^{2} / 2\right)}{t-\bar{z}}\right\} d t
$$

whence, since the expression in brackets is purely imaginary, it follows that it is.

The last conclusion of Theorem 3 is a consequence of the fact that $u(z) \exp \left(-z^{2} / 2\right)$ is the Poisson integral representation of $f(t) \exp \left(-t^{2} / 2\right)$. Hence $u(z) \exp \left(-z^{2} / 2\right) \rightarrow f(t) \exp \left(-t^{2} / 2\right)$ uniformly on compact sets and hence our conclusion.

In neither of the two cases considered so far have we looked at the harmonic conjugate. In the case of the $\left\{g_{n}\right\}$ sequence the harmonic conjugate of the function

$$
u(z)=\sum a_{n}\left(g_{n}(z)+\overline{\left.g_{n}(z)\right)}\right.
$$

will be

$$
v(z)=\sum a_{n}\left(g_{n}(z)-\overline{\left.g_{n}(z)\right)}(-i)\right.
$$


and its restriction to the real axis will be the conjugate $\widetilde{f}$ of the original $L^{2}$ function $f$. In terms of Hermite series, if $f$ has the series expansion $\Sigma a_{n} h_{n}$, then $\widetilde{f}$ is given by the series

$$
\begin{aligned}
\widetilde{f}=2 \sum_{n=0}^{\infty}\{ & \left(\sum_{k=0}^{\infty}(-1)^{n-k} \alpha_{2 n+1,2 k} a_{2 k}\right) h_{2 n+1} \\
& \left.-\left(\sum_{i=0}^{\infty}(-1)^{n-k} \alpha_{2 n, 2 k+1} a_{2 k+1}\right) h_{2 n}\right\}
\end{aligned}
$$

Clearly, since $f$ and $\Sigma a_{n} g_{n}$ are in $L^{2}$ so is $\tilde{f}$.

In the case of the series in $\left\{\widetilde{h}_{n}\right\}$ the harmonic conjugate series of

$$
u(z)=\sum \frac{i c_{n}}{2 \pi}\left[\tilde{h}_{n}(z)-\widetilde{h}_{n}(\bar{z})\right]
$$

would be

$$
v(z)=\sum \frac{c_{n}}{2 \pi}\left[\tilde{h}_{n}(z)+\tilde{h}_{n}(\bar{z})\right] .
$$

Note that the restriction of $v$ to the real axis now is not necessarily in $L^{2}$ when our original $f$ is. In fact, it is worse than that since $\widetilde{h}_{n}(x+i 0)+\widetilde{h}_{n}(x-i 0)$ is $O\left(\exp \left(x^{2} / 2\right)\right)$ as $x \rightarrow \pm \infty$. Therefore in this case we cannot in general define a conjugate series.

Our next approach will overcome the shortcomings of both of the above but will require us to alter the definition of harmonic function somewhat.

We still have not been able to imitate completely the behavior of the trigonometric functions by the Hermite functions. We next introduce a series that combines the best features of the two previous cases. Let $f$ again be in $L^{2}(-\infty, \infty)$ with Hermite series $\Sigma c_{n} h_{n}$. Then we define the two functions $u$ and $v$ by

$$
u(x, y)=\sum_{n=0}^{\infty} c_{n} \frac{h_{n}(x) \widetilde{h}_{n}(i y)}{\widetilde{h}_{n}(0)}
$$

and

$$
v(x, y)=i \sum_{n=1}^{\infty} c_{n-1} \frac{h_{n}(x) \widetilde{h}_{n}(i y)}{\widetilde{h}_{n-1}(0)}
$$

These two functions will play a role analogous to conjugate harmonic functions. Clearly $u(x, 0)=f(x)$ provided the series converges.

THEOREM 4. Let $u(x, y)$ and $v(x, y)$ be given as in (22) and (23), where $\left\{c_{n}\right\} \in l^{2}$. Then both $u$ and $v$ are real valued $C^{\infty}$ functions for $(x, y)$ in the open upper half plane which satisfy the equation $\left(\Delta^{2}-r^{2}\right) w=0$ where $r^{2}=x^{2}+y^{2}$. 
Moreover they are related by the equations

$$
(x-\partial / \partial x) u=(y-\partial / \partial y) v
$$

and

$$
(x+\partial / \partial x) v=-(y+\partial / \partial y) u
$$

We shall use the recurrence formulae for Hermite functions

$$
x h_{n}=\sqrt{n / 2} h_{n-1}+\sqrt{(n+1) / 2} h_{n+1}
$$

and

$$
h_{n}^{\prime}=\sqrt{n / 2} h_{n-1}-\sqrt{(n+1) / 2} h_{n+1},
$$

which are also satisfied by the $\tilde{h}_{n}$, to show formally that the differential equations (24) and (25) are satisfied. Indeed we see that

$$
\left(x-\frac{\partial}{\partial x}\right) u(x, y)=\sum_{n=0}^{\infty} c_{n} \sqrt{2 n+2 h_{n+1}}(x) \frac{\tilde{h}_{n}(i y)}{\widetilde{h}_{n}(0)}
$$

and

$$
\left(y-\frac{\partial}{\partial y}\right) v(x, y)=\sum_{n=1}^{\infty} c_{n-1} h_{n}(x) \frac{\sqrt{2 n} \tilde{h}_{n-1}(i y)}{\widetilde{h}_{n-1}(0)}
$$

Hence we obtain (24) and by a similar calculation (25). If we put them together we find that

$$
\begin{aligned}
\left(x+\frac{\partial}{\partial x}\right)\left(x-\frac{\partial}{\partial x}\right) u & =\left(x+\frac{\partial}{\partial x}\right)\left(y-\frac{\partial}{\partial y}\right) v=\left(y-\frac{\partial}{\partial y}\right)\left(x+\frac{\partial}{\partial x}\right) v \\
& =-\left(y-\frac{\partial}{\partial y}\right)\left(y+\frac{\partial}{\partial y}\right) u
\end{aligned}
$$

or

$$
\left(\partial^{2} / \partial x^{2}+\partial^{2} / \partial y^{2}-x^{2}-y^{2}\right) u=0 .
$$

A similar calculation holds for $v$, whence it follows that both $u$ and $v$ satisfy the partial differential equation

$$
\left(\Delta^{2}-r^{2}\right) v=0
$$

where $r^{2}=x^{2}+y^{2}$.

There are a few things we must check in order to justify these calculations. First we would like both $u$ and $v$ to be real-valued functions, and secondly we would like the series for $u$ and $v$, after two differentiations, to be uniformly convergent. 
We first observe that $h_{n}(i y)$ is real when $n$ is even and imaginary when $n$ is odd, so that $h_{n}^{2}(i y)$ is always real. Hence $\widetilde{h}_{n}(i y)=h_{n}(i y) \int_{i \infty}^{i y} 2 / h_{n}^{2}$ is real for $y>$ 0 when $n$ is odd and imaginary when $n$ is even. Now for $n=2 k$ we have

$$
\tilde{h}_{2 k}(0)=i h_{2 k}(0) \int_{\infty}^{0} 2 / h_{2 k}^{2}(i t) d t
$$

and for $n=2 k+1$ we have

$$
\begin{aligned}
\tilde{h}_{2 k+1}(0) & =\lim _{y \rightarrow 0} \frac{\int_{i \infty}^{i y} 2 / h_{n}^{2}}{1 / h_{n}(i y)}=\lim _{y \rightarrow 0} \frac{2 / h_{n}^{2}(i y)}{-h_{n}^{\prime}(i y) / h_{n}^{2}(i y)} \\
& =\lim _{y \rightarrow 0} \frac{-2}{h_{n}^{\prime}(i y)}=\frac{-2}{h_{2 k+1}^{\prime}(0)} .
\end{aligned}
$$

We may use (28) and (29) to calculate the exact values of $\tilde{h}_{n}(0)$. First by direct calculation we find that

$$
\tilde{h}_{0}(0)=(-i) \pi^{3 / 4}
$$

and that

$$
\widetilde{h}_{1}(0)=-2 / h_{1}^{\prime}(0)=-2 / \sqrt{2} h_{0}(0)=-\sqrt{2} \pi^{1 / 4}
$$

Then we use the recurrence formula

$$
\sqrt{n} / 2 \tilde{h}_{n-1}(0)+\sqrt{(n+1) / 2} \tilde{h}_{n+1}(0)=0
$$

to calculate the remaining values which are

$$
\tilde{h}_{2 k}(0)=(-i)(-1)^{k} \sqrt{\frac{2 k-1}{2 k} \cdot \frac{2 k-3}{2 k-2} \cdots \frac{1}{2} \pi^{3 / 4}}
$$

and

$$
\tilde{h}_{2 k+1}(0)=-\sqrt{2}(-1)^{k} \sqrt{\frac{2 k}{2 k+1} \cdot \frac{2 k-2}{2 k-1} \cdots \frac{2}{3}} \pi^{1 / 4} .
$$

Thus we see that both $u(x, y)$ and $v(x, y)$ are real.

To show that the series converges, let us look more closely at $\tilde{h}_{n}(i y)$. We use the fact that

$$
\tilde{h}_{n}(i y)=h_{0}^{-1}(i y) \int_{-\infty}^{\infty} \frac{h_{n}(t) h_{0}(t)}{i y-t} d t, \quad y>0, n=0,1,2, \cdots
$$

Then for any integer $p>0$, we find that 


$$
\begin{aligned}
(2 n+1)^{p} \widetilde{h}_{n}(i y) & =\int_{-\infty}^{\infty}(2 n+1)^{p} h_{n}(t) \frac{\exp \left(-y^{2} / 2\right) \exp \left(-t^{2} / 2\right)}{i y-t} d t \\
& =\int_{-\infty}^{\infty}\left(t^{2}-D_{t}^{2}\right)^{p} h_{n}(t) \frac{\exp \left(-y^{2} / 2-t^{2} / 2\right)}{i y-t} d t \\
& =\int_{-\infty}^{\infty} h_{n}(t)\left(t^{2}-D_{t}^{2}\right)^{p}\left\{\frac{\exp \left(-y^{2} / 2-t^{2} / 2\right)}{i y-t}\right\} d t \\
& =\int_{-\infty}^{\infty} h_{n}(t) \exp \left(-y^{2} / 2-t^{2} / 2\right) \frac{P_{p}(y, t)}{Q_{p}(y, t)} d t
\end{aligned}
$$

where $P_{p}$ is a polynomial in $y$ and $t$ and $Q_{p}(y, t)=(i y-t)^{2 p+1}$. Therefore the function $P_{p}(y, t) Q_{p}^{-1}(y, t) \exp \left(-t^{2} / 2\right)$ is in $L^{2}(-\infty, \infty)$ for any $y>0$, and hence by Schwarz's inequality

$$
\left|(2 n+1)^{p} \tilde{h}_{n}(i y)\right| \leqslant C_{p}(y) \exp \left(-y^{2} / 2\right)
$$

where $C_{p}(y)$ is independent of $n$ and of polynomial growth and continuous for $y>0$. This inequality allows us to deduce that the series (22) and (23) both converge uniformly for all real $x$ and $y \geqslant \epsilon>0$ provided that the coefficients $c_{n}$ are $O\left(n^{s}\right)$ for some integer $s$. The same is true for repeated partial derivatives with respect to $x$ and $y$ and multiplication by $x$ or by $y$.

Initially we assumed that the coefficients $\left\{c_{n}\right\}$ were those of an $L^{2}(-\infty, \infty)$ function. However, we could just as easily have assumed that they were the coefficients of a tempered distribution in $S^{\prime}$ and hence behaved asymptotically as $c_{n}=O\left(n^{p}\right), p \in Z$. The proof of the theorem is still valid and with a few more calculations we may prove the following theorem.

TheOREM 5. Let $f \in L^{2}(-\infty, \infty)$ (resp. $\left.S^{\prime}\right)$ with Hermite coefficients $\left\{c_{n}\right\}$. Then the function $u(x, y)$ given by (22) converges to $f$ in the sense of $L^{2}$ (resp. $\left.S^{\prime}\right)$ as $y \rightarrow 0^{+}$. The function $v(x, y)$ converges to $\widetilde{f}$ (the conjugate of $f$ ) in the sense of $L^{2}$ (resp. $S^{\prime}$ ) as $y \rightarrow 0$ where $\widetilde{f}$ is given by the series

$$
\widetilde{f}(x)=i \sum_{n=1}^{\infty} c_{n-1} \frac{\widetilde{h}_{n}(0)}{\widetilde{h}_{n-1}(0)} h_{n}(x)
$$

which is convergent in the sense of $L^{2}$ (resp. $\left.S^{\prime}\right)$.

In order to prove the theorem we need some additional simple properties of $\tilde{h}_{n}$. They are given by

LEMMA 1. Let $y \geqslant 0$; then:

(i) $\tilde{h}_{n}(i y) \tilde{h}_{n}^{-1}(0)$ is real, positive and monotonically decreasing.

(ii) $\widetilde{h}_{n}(0) \tilde{h}_{n-1}^{-1}(0)$ is bounded.

From formulas (32) and (33) we see that $\tilde{h}_{4 k+j}(0)$ and hence $\tilde{h}_{4 k+j}(i y)$ is 
imaginary negative, real negative, imaginary positive, or real positive according to whether $j=0,1,2$, or 3 respectively. Hence it is clear that

$$
\frac{d}{d y} \widetilde{h}_{n}(i y) \tilde{h}_{n}^{-1}(0)=\left(-i \sqrt{\frac{n}{2}} \widetilde{h}_{n-1}(i y)+i \sqrt{\frac{n+1}{2}} \tilde{h}_{n+1}(i y)\right) \tilde{h}_{n}^{-1}(0)
$$

is real negative. Again from (32) and (33) we see that

$$
\frac{\widetilde{h}_{2 k+1}(0)}{\widetilde{h}_{2 k}(0)}=\sqrt{\frac{2}{\pi}} i \frac{1}{\sqrt{2 k+1}} \frac{2^{2 k}(k !)^{2}}{(2 k) !} \text {. }
$$

By Stirling's formula we may find bounds on the factorials

$$
\sqrt{1 / 4 k \pi}<(2 k) ! / 2^{2 k}(k !)^{2}<\sqrt{2 / k \pi} .
$$

Hence we see that

$$
1 / 2<\left|\tilde{h}_{2 k+1}(0) / \tilde{h}_{2 k}(0)\right|<2,
$$

which gives us the desired inequality for $n$ odd. For even $n$ it suffices to use the recurrence formula for $\widetilde{h}_{n}$, namely $\sqrt{n / 2} \tilde{h}_{n-1}(0)=-\sqrt{(n+1) / 2} \tilde{h}_{n+1}(0)$ and the other inequality.

Now we return to the proof of the theorem. In the $L^{2}$ case we have

$$
\|f-u(\cdot, y)\|_{2}^{2}=\sum_{n=0}^{\infty}\left|c_{n}\right|^{2}\left(1-\tilde{h}_{n}(i y) \tilde{h}_{n}^{-1}(0)\right)^{2}
$$

where the series is dominated by $4 \Sigma\left|c_{n}\right|^{2}$ and hence converges uniformly for all $y \geqslant 0$. We can therefore take the limit as $y \rightarrow 0$ termwise and conclude that the desired result holds for the $L^{2}$ case.

The series defining $\widetilde{f}$ has coefficients which satisfy $\Sigma\left|c_{n}\right|^{2}\left|\tilde{h}_{n}(0) / \tilde{h}_{n-1}(0)\right|^{2}$ $<\infty$ and therefore we have $\tilde{f} \in L^{2}(-\infty, \infty)$ as well as the desired convergence.

In the case of $S^{\prime}$, we let $\varphi \in S$, and must show that $\langle u(\cdot, y), \varphi\rangle \rightarrow\langle f, \varphi\rangle$.

Let $d_{n}=\left\langle\varphi, h_{n}\right\rangle$; then we have

$$
\begin{aligned}
K u(\cdot, y), \varphi\rangle-\langle f, \varphi\rangle \mid \\
=\left|\sum c_{n} \bar{d}_{n}\left(1-\tilde{h}_{n}(i y) \tilde{h}_{n}^{-1}(0)\right)\right| .
\end{aligned}
$$

But the series $\Sigma c_{n} \bar{d}_{n}$ converges absolutely and by a slight modification of the $L^{2}$ argument we reach our conclusions for this case as well.

We still have not mentioned nor shown anything about pointwise convergence of $u$ and $v$ to $f$ and $\widetilde{f}$ respectively. Indeed, just as with trigonometric Fourier series, questions of pointwise convergence are much more delicate and require a more detailed look at the transformation from $f$ to $u$. This can be expressed as an integral operator:

$$
u(x, y)=\int_{-\infty}^{\infty} P(x, y, t) f(t) d t
$$


where the kernel $P(x, y, t)$ is given by

$$
P(x, y, t)=\sum_{n=0}^{\infty} h_{n}(x) \frac{\widetilde{h}_{n}(i y)}{\widetilde{h}_{n}(0)} h_{n}(t) .
$$

Similarly $v$ is related to $f$ by an integral operator with the conjugate kernel

$$
\widetilde{P}(x, y, t)=\sum_{n=0}^{\infty} h_{n+1}(x) \frac{\tilde{h}_{n+1}(i y)}{\widetilde{h}_{n}(0)} h_{n}(t) .
$$

In order to study the kernel $P(x, y, t)$ we derive first some additional properties of $\tilde{h}_{n}(i y)$.

LEMma 2. There exists a constant $C_{2}$ such that

$$
\left|\tilde{h}_{n}(i y) \tilde{h}_{n}^{-1}(0)\right| \leqslant C_{2} e^{-\sqrt{2 n+1} y}, \quad n=0,1,2, \ldots, y \geqslant 0 .
$$

The proof is based on the fact that $\left|h_{n}(i y)\right|$ is monotonically increasing with $y$ for $y \geqslant 0$. This is clear from the fact that $h_{n}(z)=H_{n}(z) \exp \left(-z^{2} / 2\right) A_{n}^{-1}$ where $H_{n}(z)$ is a polynomial with exclusively real zeros. We then use the formula, which may be derived from the differential équation for $h_{n}$, given by

$$
\begin{aligned}
h_{n}(i y)= & h_{n}(0) \cosh \sqrt{2 n+1} y+\frac{i h_{n}^{\prime}(0)}{\sqrt{2 n+1}} \sinh \sqrt{2 n+1} y \\
& +\frac{1}{\sqrt{2 n+1}} \int_{0}^{y} s^{2} h_{n}(i s) \sinh \sqrt{2 n+1}(y-s) d s .
\end{aligned}
$$

For $n$ even $h_{n}^{\prime}(0)=0$ and $h_{n}(i y)$ is real and either positive or negative for all $y \geqslant 0$. If positive then the integral is positive and $h_{n}(i y) \geqslant h_{n}(0) \cosh \sqrt{2 n+1} y$; if negative the opposite inequality holds. In either case we have

$$
\left|h_{n}(i y)\right| \geqslant\left|h_{n}(0)\right| \cosh \sqrt{2 n+1} y .
$$

For $n$ odd, $h_{n}(0)=0$ and $i h_{n}(i y)$ is real and of constant sign for all $y \geqslant 0$. If $i h_{n}(i y)>0$ then $(d / d y) i h_{n}(i y)>0$ for $y \geqslant 0$ and hence $h_{n}^{\prime}(0)<0$. Hence the same arguments hold for $n$ odd and we have

$$
\left|h_{n}(i y)\right| \geqslant\left|h_{n}^{\prime}(0) / \sqrt{2 n+1}\right| \sinh \sqrt{2 n+1} y .
$$

We now turn our attention to $\widetilde{h}_{n}(i y)$. Using the formula

$$
\widetilde{h}_{n}(i y)=h_{n}(i y) \int_{i \infty}^{i y} 2 / h_{n}^{2}
$$

we first derive the inequality

$$
\left|\tilde{h}_{n}(i y)\right| \leqslant \frac{\left|h_{n}(i y)\right|}{\left|h_{n}(i y)\right|} \int_{y}^{\infty} \frac{2 d s}{\left|h_{n}(i s)\right|} .
$$


For $n=2 k, k=0,1,2, \ldots$, we have

$$
\begin{aligned}
\left|\tilde{h}_{2 k}(i y)\right| & \leqslant \int_{y}^{\infty} \frac{2}{\left|h_{2 k}(0)\right| \cosh \sqrt{4 k+1 s}} d s \leqslant \int_{y}^{\infty} \frac{4 e^{-\sqrt{4 k+1} s}}{\left|h_{2 k}(0)\right|} d s \\
& =\frac{4 e^{-\sqrt{4 k+1} y}}{\left|h_{2 k}(0)\right| \sqrt{4 k+1}} \leqslant C_{1} \tilde{h}_{2 k}(0) e^{-\sqrt{4 k+1} y}
\end{aligned}
$$

since $h_{2 k}(0)$ and, by $(32), \widetilde{h}_{2 k}(0)$ both are greater than a constant multiple of $k^{-1 / 4}$.

In order to get a similar inequality for $\widetilde{h}_{n}$ when $n$ is odd, we first observe that $\left|h_{n}^{\prime}(i y)\right|$ is also monotonically increasing with $y$ for $y \geqslant 0$. Therefore we have

$$
\left|\widetilde{h}_{n}(i y)\right|=\left|\frac{h_{n}(i y)}{h_{n}^{\prime}(i y)} h_{n}^{\prime}(i y) \int_{i \infty}^{i y} \frac{2}{h_{n}^{2}}\right| \leqslant\left|\frac{h_{n}(i y)}{h_{n}^{\prime}(i y)}\right|\left|\int_{i \infty}^{i y} \frac{2 h_{n}^{\prime}}{h_{n}^{2}}\right|=\frac{2}{\left|h_{n}^{\prime}(i y)\right|} .
$$

But by differentiating the expression for $h_{n}(i y)$, we obtain, for odd $n$,

$$
\begin{aligned}
h_{n}^{\prime}(i y) & =-i(d / d y) h_{n}(i y) \\
& =h_{n}^{\prime}(0) \cosh \sqrt{2 n+1} y+(-i) \int_{0}^{y} s^{2} h_{n}(i s) \cosh \sqrt{2 n+1}(y-s) d s
\end{aligned}
$$

in which both terms on the right are real and have the same sign. Hence we have

$$
\left|h_{n}^{\prime}(i y)\right| \geqslant\left|h_{n}^{\prime}(0)\right| \cosh \sqrt{2 n+1 y}
$$

and therefore

$$
\left|\tilde{h}_{n}(i y)\right| \leqslant\left(4 /\left|h_{n}^{\prime}(0)\right|\right) e^{-\sqrt{2 n+1} y}, \quad n=1,3,5, \ldots .
$$

Now since $h_{n}^{\prime}(0)=\sqrt{2 n} h_{n-1}(0)$, we see that $\left|\tilde{h}_{n}(0) h_{n}^{\prime}(0)\right|^{-1}$ is bounded and hence reach our desired conclusion for odd $n$ as well.

We must refine the inequality in Lemma 2 somewhat in order to derive some needed properties of the kernel $P(t, x, y)$.

LEMMA 3. There exists a cubic polynomial $P(y)$ such that

$$
\left|\frac{\tilde{h}_{n}(i y)}{\widetilde{h}_{n}(0)}-e^{-\sqrt{2 n+1} y}\right| \leqslant \frac{P(y) e^{-\sqrt{2 n+1} y}}{\sqrt{2 n+1}}, \quad y \geqslant 0, n=0,1,2, \ldots
$$

In order to prove this lemma we use the fact that $\tilde{h}_{n}$ satisfies the same differential equation as $h_{n}$ and therefore that

$$
\begin{aligned}
\frac{\widetilde{h}_{n}(i y)}{\widetilde{h}_{n}(0)}= & \cosh \sqrt{2 n+1} y+i \frac{\widetilde{h}_{n}^{\prime}(0)}{\widetilde{h}_{n}(0) \sqrt{2 n+1}} \sinh \sqrt{2 n+1 y} \\
& +\frac{1}{\sqrt{2 n+1}} \int_{0}^{y} s^{2} \frac{\widetilde{h}_{n}(i s)}{\widetilde{h}_{n}(0)} \sinh \sqrt{2 n+1}(y-s) d s .
\end{aligned}
$$


We know from Lemma 2 that $\tilde{h}_{n}(i y) \rightarrow 0$ as $y \rightarrow \infty$. Therefore the difference

$$
\begin{aligned}
\frac{\tilde{h}_{n}(i y)}{\widetilde{h}_{n}(0)}-e^{-\sqrt{2 n+1} y} & \\
= & {\left[1+\frac{i \widetilde{h}_{n}^{\prime}(0)}{\sqrt{2 n+1} \widetilde{h}_{n}(0)}\right] \sinh \sqrt{2 n+1 y} } \\
& +\frac{1}{\sqrt{2 n+1}} \int_{0}^{y} s^{2} \frac{\widetilde{h}_{n}(i s)}{\widetilde{h}_{n}(0)} \frac{e^{\sqrt{2 n+1}(y-s)}-e^{-\sqrt{2 n+1}(y-s)}}{2} d s
\end{aligned}
$$

also converges to 0 as $y \rightarrow \infty$. Since the terms

$$
e^{-\sqrt{2 n+1} y} \text { and } \int_{0}^{y} s^{2} \tilde{h}_{n}(i s) e^{-\sqrt{2 n+1}(y-s)} d s
$$

clearly do the same, it follows that the remaining terms converge to 0 as $y \rightarrow \infty$. These remaining terms may be expressed as

$$
\left[1+\frac{i \tilde{h}_{n}^{\prime}(0)}{\sqrt{2 n+1} \widetilde{h}_{n}(0)}+\frac{1}{\sqrt{2 n+1}} \int_{0}^{y} s^{2} \frac{\widetilde{h}_{n}(i s)}{\widetilde{h}_{n}(0)} e^{-\sqrt{2 n+1} s} d s\right] \frac{e^{\sqrt{2 n+1} y}}{2},
$$

whence it follows that the factor in brackets also converges to 0 as $y \rightarrow \infty$ and hence is equal to

$$
-\frac{1}{\sqrt{2 n+1}} \int_{y}^{\infty} s^{2} \frac{\widetilde{h}_{n}(i s)}{\widetilde{h}_{n}(0)} e^{-\sqrt{2 n+1} s} d s .
$$

By combining the various terms in (46) we obtain the inequality

$$
\begin{aligned}
\left|\frac{\widetilde{h}_{n}(i y)}{\widetilde{h}_{n}(0)}-e^{-\sqrt{2 n+1} y}\right| \\
\leqslant\left|1+\frac{i \widetilde{h}_{n}^{\prime}(0)}{\sqrt{2 n+1} \widetilde{h}_{n}(0)}+\int_{0}^{y} \frac{s^{2} \widetilde{h}_{n}(i s)}{\sqrt{2 n+1 \tilde{h}_{n}(0)}} e^{\sqrt{2 n+1} s} d s\right| \frac{e^{-\sqrt{2 n+1} y}}{2} \\
+\int_{y}^{\infty} \frac{s^{2} \widetilde{h}_{n}(i s)}{\sqrt{2 n+1} \widetilde{h}_{n}(0)} e^{-\sqrt{2 n+1} s} d s \frac{e^{\sqrt{2 n+1} y}}{2} .
\end{aligned}
$$

We now use (32) and (33) to estimate $i \tilde{h}_{n}^{\prime}(0) / \tilde{h}_{n}(0) \sqrt{2 n+1}$.

By using the fact that $\widetilde{h}_{n}^{\prime}(0)=-\sqrt{2 n+2} \widetilde{h}_{n+1}(0)$ and Stirling's formula, we see that for $n=2 k$, 


$$
\begin{aligned}
& -\frac{i \tilde{h}_{2 k}^{\prime}(0)}{\widetilde{h}_{2 k}(0) \sqrt{4 k+1}}=\frac{(-i)\left(-\sqrt{4 k+2)} \tilde{h}_{2 k+1}(0)\right.}{\tilde{h}_{2 k}(0) \sqrt{4 k+1}} \\
& =\frac{(-i)(-\sqrt{4 k+2})(-\sqrt{2})(-1)^{k} \sqrt{\frac{2 k}{2 k+1} \cdot \frac{2 k-2}{2 k-1} \cdots \frac{2}{3}} \pi^{1 / 4}}{(-i)(-1)^{k} \sqrt{4 k+1} \sqrt{\frac{2 k-1}{2 k} \cdot \frac{2 k-3}{2 k-2} \cdots \frac{1}{2}} \pi^{3 / 4}} \\
& =2 \cdot \frac{2 k}{2 k-1} \cdot \frac{2 k-2}{2 k-3} \cdots \frac{2}{1} \frac{1}{\pi^{1 / 2} \sqrt{4 k+1}} \\
& =\frac{2}{\pi^{1 / 2} \sqrt{4 k+1}} \frac{2^{2 k}[k !]^{2}}{[2 k] !} \approx \frac{2}{\pi^{1 / 2} \sqrt{4 k+1}} \frac{2^{2 k} k^{2 k} e^{-2 k}}{\sqrt{4 k \pi}(2 k)^{2 k} e^{-2 k}} 2 k \pi \\
& =\sqrt{4 k /(4 k+1}) .
\end{aligned}
$$

Similarly for $n=2 k+1$, we obtain

$$
\begin{aligned}
& \frac{-i \tilde{h}_{2 k+1}^{\prime}(0)}{\widetilde{h}_{2 k+1}(0) \sqrt{4 k+3}}=(-i) \sqrt{4 k+1} \tilde{h}_{2 k}(0) \\
& =\frac{(-i) \sqrt{4 k+2}(-i)(-1)^{k} \sqrt{\frac{2 k-1}{2 k} \cdots \frac{1}{2}} \pi^{3 / 4}}{\sqrt{4 k+3}(-2)(-1)^{k} \sqrt{\frac{2 k}{2 k+1} \cdots \frac{2}{3}} \pi^{1 / 4}} \\
& =\frac{\sqrt{2 k+1} \sqrt{4 k+2}}{\sqrt{4 k+3}} \frac{2 k-1}{2 k} \cdots \frac{1}{2} \sqrt{\frac{\pi}{2}}=\frac{\sqrt{2 k+1} \sqrt{4 k+2}(2 k) !}{\sqrt{4 k+32^{2 k}(k !)^{2}}} \sqrt{\frac{\pi}{2}} \\
& \approx \frac{\sqrt{2 k+1} \sqrt{4 k+2}}{\sqrt{4 k+3}} \frac{\sqrt{4 k \pi}}{2 k \pi} \sqrt{\frac{\pi}{2}}=\frac{2 k+1}{\sqrt{4 k+3} \sqrt{k}} \cdot
\end{aligned}
$$

We now return to our basic inequality (47). We have all we need to prove the inequality in the lemma. It is completed by using Lemma 2 and the asymptotic expressions (48) and (49) in (47). The last term in (47) is estimated by using integration by parts.

We are now in position to derive the properties we shall need about the kernel $P(t, x, y)$ in order to prove a pointwise convergence theorem. Fortunately much of the difficult work has been done.

We first decompose $P(t, x, y)$ into the sum of two kernels one of which is of positive type and the other of which is nonsingular. 


$$
\begin{aligned}
P(t, x, y) & =\sum h_{n}(t) h_{n}(x) \widetilde{h}_{n}(i y) / \widetilde{h}_{n}(0) \\
& =\sum h_{n}(t) h_{n}(x) e^{-\sqrt{2 n+1} y}+\sum h_{n}(t) h_{n}(x) e_{n}(y) \\
& =P_{1}(t, x, y)+R(t, x, y) .
\end{aligned}
$$

Since by Lemma 3 , we have $\left|e_{n}(y)\right| \leqslant C_{3}(2 n+1)^{-1 / 2}$ and since $\left|h_{n}(x)\right| \leqslant$ $C(x)(2 n+1)^{-1 / 4}$, it is clear that $R(t, x, y) \in L^{2}(-\infty, \infty)$ as a function of $t$ for each $(x, y)$ in the upper half plane. Also since $e_{n}(0)=0$

$$
\lim _{y \rightarrow 0^{+}} \int_{-\infty}^{\infty}|R(t, x, y)|^{2} d t=\sum h_{n}^{2}(x) \lim _{y \rightarrow 0^{+}}\left|e_{n}^{2}(y)\right|=0
$$

which is valid because of the uniform convergence of the series. Now the kernel $\sum h_{n}(t) h_{n}(x) e^{-\sqrt{2 n+1} y}=P_{1}(t, x, y)$ is essentially the same as the kernel considered by Muckenhoupt $[\mathrm{M}]$, which except for a factor $\exp \left(-x^{2} / 2-y^{2} / 2\right)$ is given by $\Sigma h_{n}(t) h_{n}(x) e^{-\sqrt{2 n} y}=P_{2}(t, x, y)$. He shows (p. 237) that if $f \in$ $L^{P}(-\infty, \infty), 1 \leqslant P \leqslant \infty$, then

$$
\int_{-\infty}^{\infty} P_{2}(t, x, y) f(t) d t \rightarrow f(x) \quad \text { a.e. as } y \rightarrow 0^{+} \text {. }
$$

This implies the same result for $P_{1}$ in place of $P_{2}$ since $P_{1}-P_{2}$ is a continuous function for all $t, x \in R^{1}$ and $y \geqslant 0$ and

$$
\lim _{y \rightarrow 0^{+}}\left\|P_{1}(t, x, y)-P_{2}(t, x, y)\right\|_{2}=0
$$

by the same argument used for $R$.

We now can put together the pieces of the puzzle to give us a pointwise convergence theorem.

THEOREM 6. Let $f \in L^{2}(-\infty, \infty)$. Then the function $u(x, y)$ given by (22) converges to $f(x)$ a.e. as $y \rightarrow 0^{+}$.

The proof uses the observations we made previously:

$$
\begin{aligned}
u(x, y)= & \int_{-\infty}^{\infty} P(t, x, y) f(t) d t \\
& =\int_{-\infty}^{\infty} P_{2}(t, x, y) f(t) d t+\int_{-\infty}^{\infty}\left\{P_{1}(t, x, y)-P_{2}(t, x, y)\right\} f(t) d t \\
& +\int_{-\infty}^{\infty} R(t, x, y) f(t) d t \rightarrow f(x)+0+0 \quad \text { a.e. as } y \rightarrow 0^{+} .
\end{aligned}
$$

There are still lots of loose ends to pick up. We have not looked at $L^{P}$ convergence and pointwise convergence for $L^{P}$ functions. Nor for that matter have we discussed the local behavior of the conjugate function $v(x, y)$. 
By analogy with the theory of functions of a complex variable one can form functions of the complex variable $z=x+i y$ in upper half plane by combining $u$ and $v$ :

$$
f(z)=u(z)+i v(z)=u(x, y)+i v(x, y)
$$

Many properties of holomorphic functions carry over in modified form to the kind of functions we have. These have been studied by Vekua (see [V]) in a more general framework.

\section{REFERENCES}

[S] G. Szegठ, Orthogonal polynomials, rev. ed., Amer. Math. Soc. Colloq. Publ., vol. 23, Amer. Math. Soc., Providence, R. I., 1959. MR 21 \#5029.

[V] I. M. Vekua, Generalized analytic functions, Fizmatgiz, Moscow, 1959; English transl., Pergamon Press, London; Addison-Wesley, Reading, Mass., 1962. MR 21 \#7288; 27 \#321.

[W] G. G. Walter, Hermite series solutions of differential equations, J. Differential Equations 10 (1971), 1-16. MR 43 \#3801.

[M] B. Muckenhoupt, Poisson integrals for Hermite and Laguerre expansions, Trans. Amer. Math. Soc. 139 (1969), 231-242. MR 40 \#3158.

DEPARTMENT OF MATHEMATICS, UNIVERSITY OF WISCONSIN-MILWAUKEE, MILWAUKEE, WISCONSIN 53201 